\title{
Inhibition of induced autophagy increases apoptosis of Nara-H cells
}

\author{
OSAMU NAKAMURA ${ }^{1}$, TOSHIAKI HITORA ${ }^{1}$, TOSHIHIRO AKISUE ${ }^{2}$, \\ TERUYA KAWAMOTO $^{2}$, YOSHIKI YAMAGAMI ${ }^{1}$ and TETSUJI YAMAMOTO ${ }^{1}$ \\ ${ }^{1}$ Department of Orthopaedic Surgery, Kagawa University School of Medicine, Miki-cho, Kita-gun, Kagawa; \\ ${ }^{2}$ Department of Orthopaedic Surgery, Kobe University Graduate School of Medicine, Kobe, Hyogo, Japan
}

Received June 9, 2011; Accepted July 15, 2011

DOI: $10.3892 /$ ijo.2011.1148

\begin{abstract}
Inhibition of the mTOR signaling pathway promotes initiation of autophagy. However, recent studies indicate that autophagy is a self-defense mechanism of cancer cells that are subjected to anti-tumor agents and that blocking autophagy can trigger apoptosis. Here, we examined the effects of an mTOR inhibitor, temsirolimus, on a malignant fibrous histiocytoma (MFH) cell line, Nara-H cells, and the effect of suppressing autophagy on the induction of apoptosis in these MFH cells. In Nara-H cells, we examined the effects of temsirolimus treatment on cell proliferation using the CellTiter $96^{\circledR}$ AQueous One Solution Cell Proliferation Assay and on phosphorylation of mTOR pathway components and autophagy using Western blot-based assays. Furthermore, we examined the effects of temsirolimus with or without 3-methyladenine (3-MA) on induction of apoptosis using fluorescent microscopic analysis. In Nara-H cells, temsirolimus treatment inhibited cell proliferation, suppressed phosphorylation of mTOR pathway components, and induced autophagy as assessed by LC-3 II expression. Moreover, treatment with a combination of temsirolimus and 3-MA induced apoptosis in Nara-H cells. Apparently, simultaneous inhibition of autophagy and mTOR induced apoptosis in Nara-H cells because inhibition of autophagy prevented the cells from protecting themselves from the effects of the inhibition of mTOR. Therefore, a combination therapy that includes an mTOR inhibitor and an autophagy inhibitor (temsirolimus and 3-MA, respectively) may effectively treat MFH by inducing apoptosis in tumor cells.
\end{abstract}

\section{Introduction}

Malignant fibrous histiocytoma (MFH) is one of the most common soft tissue sarcomas in human adults (1). Advances in treatment of MFH have led to multimodal treatments that may include surgery, chemotherapy, and/or radiation therapy. These treatments have resulted in significant improvements in quality

Correspondence to: Dr Toshiaki Hitora, Department of Orthopaedic Surgery, Kagawa University, 1750-1 Ikenobe, Miki-cho, Kita-gun, Kagawa 761-0793, Japan

E-mail: thitora@med.kagawa-u.ac.jp

Key words: autophagy, apoptosis, mTOR, temsirolimus of life for patients with soft tissue sarcomas. However, the present chemotherapeutic protocols for some of these sarcoma are not as effective as those for other malignancies, and the prognosis for some patients with sarcoma is poor due to local tumor recurrence and metastases (2). Developing new strategies for assessing prognosis, overcoming resistance to anticancer therapies, and preventing or treating metastasis are important goals in the treatment of patients with sarcoma (3).

In recent years, drugs that target specific molecules have been developed as treatments for other human malignancies, including cancers and sarcoma. These drugs often selectively inhibit specific molecules, such as growth factor receptors or intracellular signaling proteins that are related to tumor proliferation, migration, and/or metastasis. Here, we focused on the mammalian target of rapamycin (mTOR) signaling pathway.

The mTOR protein is an essential serine/threonine kinase that belongs to the phosphoinositide-3-OH kinase (PI3K)-related kinase family (4). It is an intracellular protein that mediates cell growth, proliferation, migration, and survival and promotes angiogenesis in many cancer types $(5,6)$. Inhibitors of mTOR have antitumor effects in many human malignancies $(7,8)$. Inhibition of mTOR affects pathway-mediated transcription and translation, leading to cell cycle arrest and anti-angiogenesis. Regarding the anti-tumor mechanism of mTOR inhibitors, some studies indicate that inhibitors of Akt and its downstream target, mTOR signaling, have anti-tumor effects because inhibition of this pathway contributes to the initiation of autophagy (9-11).

Autophagy has recently gained attention because of its paradoxical roles in cell survival and cell death, particularly in the pathogenesis and treatment of cancer (12). Regulation of autophagy is highly complex with inputs from the cellular environment through the PI3K/Akt/mTOR pathway (13). Not surprisingly, there is an intricate relationship between autophagy and apoptosis. Recent studies indicate that autophagy can function as a self-defense mechanism in cells that are subjected to anti-tumor agents and that blocking autophagy can trigger activation of apoptosis (14-16). Based on these finding, it has been suggested that inhibitors of autophagy, such as 3-methyladenine (3-MA), might be an effective treatment for $\mathrm{MFH}$ because they activate apoptosis.

Recent studies demonstrate that 3-MA, a commonly-used inhibitor of autophagy, promotes chemotherapeutic druginduced apoptosis in cancer cells. Reportedly, 3-MA inhibits the activity of PI3K and blocks the formation of pre-autophagosomes, autophagosomes, and autophagic vacuoles (17). 
The purpose of this study was to examine the effects of the mTOR inhibitor, temsirolimus, on Nara-H cells (an MFH-derived cell line). We examined whether temsirolimus affected suppression of phosphorylation of proteins in the mTOR pathway and/ or induction of autophagy in Nara-H cells. Furthermore, we examined whether a combination of temsirolimus and 3-MA induced apoptosis in Nara-H cells.

\section{Materials and methods}

Chemical reagents. Temsirolimus (CCI-779) was purchased from BioVision Research (Linda Vista, Mountain View, CA, USA), dissolved in dimethyl sulfoxide (DMSO), and stored at $-20^{\circ} \mathrm{C}$. The inhibitor of autophagy, 3-MA, was purchased from Sigma Chemical Co. (St. Louis, MO, USA), dissolved in dimethylformamid (DMF, $1 \mathrm{mg} / \mathrm{ml}$ ), and stored at room temperature.

Cell lines and cell culture. Nara-H cells were purchased from ScienStuff Co. (Nara, Japan). The Nara-H cell line was established from a myxoid MFH of the uterus by Kiyozuka et al (18). These cells were grown in Dulbecco's modified Eagle's medium (DMEM; Sigma-Aldrich, St. Louis, MO, USA) containing $10 \%$ fetal bovine serum (FBS; Sigma-Aldrich) and $100 \mathrm{U} / \mathrm{ml}$ penicillin. These cells were routinely maintained at $37^{\circ} \mathrm{C}$ in a humidified $5 \% \mathrm{CO}_{2}$ atmosphere, and cultures were used at mid-log phase. Cells were divided into four groups, control (no inhibitor), tem (temsirolimus), 3-MA, tem plus 3-MA (temsirolimus + 3-MA), for the autophagy and apoptosis assays. Cells were seeded onto culture dishes and cultured in growth medium for $48 \mathrm{~h}$, and then the growth medium was replaced with fresh medium with or with out inhibitors.

In vitro proliferation assay. Cell proliferation was determined by CellTiter $96^{\circledR}$ AQueous One Solution Cell Proliferation Assay (Promega, Madison, WI, USA). Cells were trypsinized and seeded at a density of $1 \times 10^{4}$ cells/well in 96-well cell culture plates with $200 \mu 1$ culture medium containing $10 \%$ FBS and incubated for $48 \mathrm{~h}$. Following this initial incubation, the growth medium was replaced with medium containing $10 \%$ FBS and temsirolimus at a concentration of $0,1,2.5,5$, $10,25,50$, or $100 \mu \mathrm{M}$. After 24,36 or $48 \mathrm{~h}$, the medium was removed, the cells were washed with phosphate-buffered saline (PBS), fresh medium containing 3-(4,5-dimethylthiazol-2-yl)5-(3-carboxymethoxyphenyl)-2-(4-sulfophenyl)-2H-tetrazolium (MTS) reagent (100 $\mu 1$ medium plus $20 \mu 1 \mathrm{MTS}$ regent/well) was added to each well. In the experiments testing the combined effect of temsirolimus and 3-MA, cells were pretreated with $5 \mathrm{mM}$ 3-MA for $90 \mathrm{~min}$ before temsirolimus was added to the culture medium, and then cells were treated with $25 \mu \mathrm{M}$ temsirolimus with $5 \mathrm{mM} 3$-MA for $48 \mathrm{~h}$. In experiments testing the effect of temsirolimus or 3-MA, cells were treated with $25 \mu \mathrm{M}$ temsirolimus or $5 \mathrm{mM} 3-\mathrm{MA}$ for $24 \mathrm{~h}$. The optical density was measured at $490 \mathrm{~nm}$ using an automatic microplate reader after $2 \mathrm{~h}$ of further incubation following the addition of the MTS reagent. Absorbency is directly proportional to the number of living cells. The percent viability of each well was calculated. At least three independent experiments were performed.

Western blot analyses. Cells were trypsinized and seeded at a density of $6 \times 10^{5}$ cells/well in 6 -well cell culture plates in $2 \mathrm{ml}$ culture medium with $10 \%$ FBS. After 48 h, cells were treated with $10 \%$ FBS contain temsirolimus at the indicated time and concentration. In the experiments testing the combined effect of temsirolimus and 3-MA, cells were pretreated with $5 \mathrm{mM}$ 3-MA for $90 \mathrm{~min}$ before temsirolimus was added to the culture medium, and then cells were treated with $35 \mu \mathrm{M}$ temsirolimus with $5 \mathrm{mM}$ 3-MA for $24 \mathrm{~h}$. In experiments testing the effect of temsirolimus or 3-MA, cells were treated with $35 \mu \mathrm{M}$ temsirolimus or $5 \mathrm{mM} 3-\mathrm{MA}$ for $24 \mathrm{~h}$. Following treatment, the culture medium was replaced with lysis buffer (Cell Signalling Technology, Berverly, MA, USA), and cells were lysed on ice for $20 \mathrm{~min}$. The cell lysates were spun at 15,000 x g using the Tomy MTX-150 centrifuge (Tomy Seiko Co., Ltd., Fukuoka, Japan) at $4^{\circ} \mathrm{C}$ for $30 \mathrm{~min}$. The supernatant was then collected as the total cellular protein extracts. The protein concentrations were determined by Protein Assay Bicinchoninate kit (Nacalai Tesque, Inc., Kyoto, Japan) and standardized with bovine serum albumin. The samples of total cellular protein were loaded onto an SDS polyacrylamide gel $(7.5,10$, or $12 \%$ commercial precast gel, Wako, Tokyo, Japan), and the proteins were separated by SDS-PAGE under reducing conditions. The mTOR and phospho-mTOR proteins were separated on a $7.5 \%$ SDS gel; the Akt, phospho-Akt, p70 ribosomal protein S6 kinase (p70S6K), phospho-p70S6K, $\alpha$-tublin, poly-(ADP-ribose) polymerase (PARP) proteins were separated on 10\% SDS gel; and 4-E-binding protein 1 (4E-BP1), phospho-4E-BP1, and microtubule-associated protein light-chain 3 (LC-3) proteins were separated on a $12 \%$ SDS gel. The separated proteins were electrophoretically transferred to nitrocellose membranes (GE Healthcare Bio-Sciences, Piscataway, NJ, USA). The membranes were blocked for $90 \mathrm{~min}$ in the blocking buffer containing Trisbuffered saline (TBS-T) and 10\% EZ block (Atto Co., Tokyo, Japan). Then, the membranes were incubated with primary antibodies which diluted in the blocking buffer overnight at $4^{\circ} \mathrm{C}$. Antibodies for Akt, phospho-Akt (Thr 308) were purchased from Santa Cruz Biotechnology, Inc. (Santa Cruz, CA, USA). Anti-mTOR and anti-phospho-mTOR (Ser 2448) antibodies were purchased from R\&D Systems (Minneapolis, MN, USA). Anti-4E-BP1, anti-phospho-4E-BP1 (Ser 65), anti-p70S6K, and anti-phospho-p70S6K (Thr421/Ser424) antibodies were purchased from Cell Signaling Technology (Beverly, MA, USA), and anti- $\alpha$-tublin antibody was purchased from Sigma. Anti-PARP antibody was purchased from BD Biosciences (Franklin Lakes, NJ, USA). Anti-LC-3 antibody was purchased from MBL Co. (Nagoya, Japan). These primary antibodies are listed and characterized in Table I.

The specific HRP-conjugated secondary antibody incubations were performed overnight at $4^{\circ} \mathrm{C}$ with gentle agitation. Bound antibodies were detected using the ECL plus Western blotting detection system (GE Healthcare Bio-Sciences) and LAS-1000 plus image analyzer (Fuji Film Co., Tokyo, Japan). Specific signals were quantified by densitometric analysis (Image J software).

Cell cycle fraction by flow cytometry. Cells were stained with propidium iodide (PI) to assess the cell cycle phase during fraction by flow cytometry. Cells were seeded at a density of $6 \times 10^{5}$ and cultured for $48 \mathrm{~h}$. In the experiments testing the combined effect of temsirolimus and 3-MA, cells were pretreated with $5 \mathrm{mM}$ 3-MA for $90 \mathrm{~min}$ before temsirolimus was added to the 
Table I. Primary antibodies used in Western blot analysis.

\begin{tabular}{lllll}
\hline Target & \multicolumn{1}{c}{ Souce } & Host & Dilution & Second antibody \\
\hline Akt & St. Cruz Biot. & Rabbit & $1: 1000$ & Anti-rabbit \\
Phospho-Akt & St. Cruz Biot. & Rabbit & $1: 1000$ & Anti-rabbit \\
mTOR & R\&D Systems & Rabbit & $1: 1000$ & Anti-rabbit \\
Phospho-mTOR & R\&D Systems & Rabbit & $1: 1000$ & Anti-rabbit \\
4E-BP1 & Cell Signalings & Rabbit & $1: 1000$ & Anti-rabbit \\
Phospho-4E-BP1 & Cell Signalings & Rabbit & $1: 1000$ & Anti-rabbit \\
p70S6 & Cell Signalings & Rabbit & $1: 1000$ & Anti-rabbit \\
Phospho-p70S6 & Cell Signalings & Rabbit & $1: 1000$ & Anti-rabbit \\
PARP & BD Biosciences & Mouse & $1: 1000$ & Anti-mouse \\
LC-3 & MBL & Rabbit & $1: 1000$ & Anti-rabbit \\
$\alpha$-tublin & Sigma & Mouse & $1: 1000$ & Anti-mouse \\
\hline
\end{tabular}

culture medium, and then cells were treated with $35 \mu \mathrm{M}$ temsirolimus with $5 \mathrm{mM} 3$-MA for $24 \mathrm{~h}$. In experiments testing the effect of temsirolimus or 3-MA, cells were treated with $35 \mu \mathrm{M}$ temsirolimus or $5 \mathrm{mM} 3-\mathrm{MA}$ for $24 \mathrm{~h}$. Then cells were washed gently three times in PBS, fixed with $70 \%$ ethanol, and stored at $-30^{\circ} \mathrm{C}$. The next day, the samples were washed three times in PBS and incubated with $10 \mu \mathrm{M}$ RNase, DNase-free (Roche Applied Science, Penzberg, Germany) and $50 \mu \mathrm{M}$ PI (Miltenyi Biotec Inc., Auburn, CA, USA) for $20 \mathrm{~min}$ at $37^{\circ} \mathrm{C}$. Cell-cycle analysis was performed using a Flow Cytometer (FC-500, Beckman Coulter, Inc., Brea, CA, USA) for evaluation of the sub- $\mathrm{G}_{1}$ fraction. Data are representative of 3 independent experiments.

Determination of apoptosis by Annexin V-FITC and PI stain analysis. Cells were trypsinized and seeded at a density of $6 \times 10^{5}$ cells/well in 6-well cell culture plates in $2 \mathrm{ml}$ culture medium with $10 \% \mathrm{FBS}$ and then cultured for $48 \mathrm{~h}$. In the experiments testing the combined effect of temsirolimus and 3-MA, cells were pretreated with $5 \mathrm{mM} 3$-MA for $90 \mathrm{~min}$ before temsirolimus was added to the culture medium, and then cells were treated with $35 \mu \mathrm{M}$ temsirolimus with $5 \mathrm{mM}$ 3-MA for $24 \mathrm{~h}$. In experiments testing the effect of temsirolimus or 3-MA, cells were treated with $35 \mu \mathrm{M}$ temsirolimus or $5 \mathrm{mM} 3-\mathrm{MA}$ for $24 \mathrm{~h}$. Cells were then incubated with Annexin V-FITC and PI using Annexin-V-Fluos Staining Kit (Roche Applied Science) for $15 \mathrm{~min}$ in a dark room according to the manufacturer's recommendations. Stained cells were observed under a fluorescence microscope (Keyence Co., Osaka, Japan) equipped with a filter system (DAPI-BP for Annexin V: excitation wavelength $377 \mathrm{~nm}$ and detection $447 \mathrm{~nm}$, TRITC for PI: excitation wavelength $543 \mathrm{~nm}$ and detection $593 \mathrm{~nm}$ ). To quantify Annexin-V and PI incorporation, at least 100 cells from each treatment group were examined under fluorescence microscopy, and the percentage of Annexin V-positive or Annexin V-plus-PI positive cells were calculated. The 100 cells sampled were chosen randomly to avoid bias.

Detection of autophagic vacuoles with monodansylcadaverine. Autophagic vacuoles were detected with monodansylcadaverine (MDC) by incubating cells with MDC solution (1:1000 in Cell Based Assay Buffer, $50 \mu \mathrm{M}$ ) in PBS using Autophagy/ Cytotoxicity Dural Staining Kit (Cayman Chemical Co., MI,
USA). Cells were seeded at a density of $6 \times 10^{5}$ cells/well in 6-well cell culture plates in $2 \mathrm{ml}$ culture medium with $10 \% \mathrm{FBS}$ and then cultured for $48 \mathrm{~h}$. In the experiments testing the combined effect of temsirolimus and 3-MA, cells were pretreated with 3-MA for $90 \mathrm{~min}$ before temsirolimus was added to the culture medium, and then cells were treated with $35 \mu \mathrm{M}$ temsirolimus with $5 \mathrm{mM}$ 3-MA for $24 \mathrm{~h}$. In experiments testing the effect of temsirolimus or 3-MA, cells were treated with $35 \mu \mathrm{M}$ temsirolimus or $5 \mathrm{mM}$ 3-MA for $24 \mathrm{~h}$. Cells were then incubated with MDC for $15 \mathrm{~min}$ and immediately analyzed by fluorescence microscope (DMI 4000 B, Leica Microsystems, Wetzlar, Germany) using a fluorescence microscope equipped with a filter system (excitation wavelength of 460-500 nm, emission wavelength of 512-542 nm). Bright-field and fluorescence images were merged. To quantify MDC incorporation, at least 100 cells from each treatment group were examined via fluorescence microscopy, and the percentage of MDC-positive cells was calculated. The 100 cells sampled were chosen randomly to avoid bias.

Statistical analysis. All data and results presented are representative of, or calculated from, at least three independent experiments. For the cell proliferation assay, flow cytometry, and morphological examination, differences between treatment groups were determined with the StatView for Windows software package. The data collected in three independent experiments for each group are expressed as mean \pm standard deviation (SD) and analyzed statically using ANOVA with Fisher's PLSD post $h o c$ test. A $\mathrm{p}<0.05$ was regarded as statistically significant.

\section{Results}

Temsirolimus inhibited cell proliferation. We examined the effect of temsirolimus on Nara-H cell proliferation using the CellTiter 96 AQueous One Solution Cell Proliferation Assay to determine whether temsirolimus inhibited cell proliferation. Temsirolimus inhibited Nara-H cell proliferation in a doseand time-dependent manner. The $\mathrm{IC}_{50}$ for $48 \mathrm{~h}$ of temsirolimus treatment in Nara-H cell was $25.86 \pm 2.36 \mu \mathrm{M}$ (Fig. 1A).

We then examined the effect of temsirolimus with or without 3-MA on Nara-H cell proliferation. Based on the $\mathrm{IC}_{50}$ of temsirolimus after $48 \mathrm{~h}$, we examine proliferation of Nara-H cells treated with $25 \mu \mathrm{M}$ Temsirolimus (tem group), $5 \mathrm{mM}$ 3-MA (3-MA 

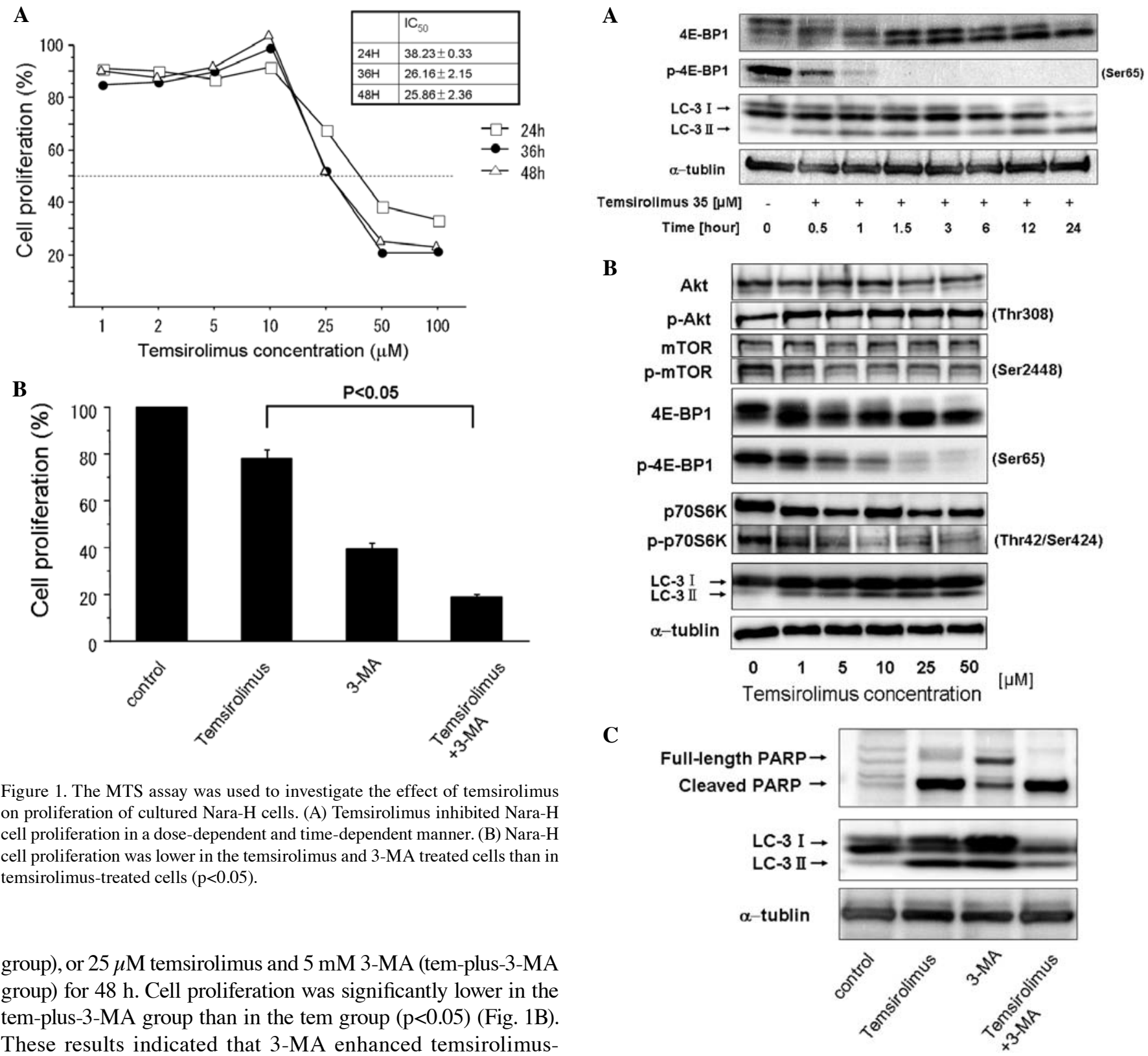

Figure 1. The MTS assay was used to investigate the effect of temsirolimus on proliferation of cultured Nara-H cells. (A) Temsirolimus inhibited Nara-H cell proliferation in a dose-dependent and time-dependent manner. (B) Nara- $\mathrm{H}$ cell proliferation was lower in the temsirolimus and 3-MA treated cells than in temsirolimus-treated cells $(\mathrm{p}<0.05)$.

group), or $25 \mu \mathrm{M}$ temsirolimus and $5 \mathrm{mM}$ 3-MA (tem-plus-3-MA group) for $48 \mathrm{~h}$. Cell proliferation was significantly lower in the tem-plus-3-MA group than in the tem group $(\mathrm{p}<0.05)$ (Fig. 1B). These results indicated that 3-MA enhanced temsirolimusmediated suppression of Nara-H cell proliferation.

Inhibitory effects on the expression of Akt/mTOR pathway. Western blot analyses demonstrated that temsirolimus and temsirolimus-plus-3-MA treatments promoted phosphorylation of key components in the Akt-mTOR pathway. We examined the time course of 4E-BP1, phospho-4E-BP1, and LC-3 expression in treated and control Nara-H cells. These proteins are downstream of mTOR, and LC-3, LC-3II (16 kDa) in particular, is the first protein to specifically localize to autophagosome membranes. Therefore, LC-3II expression can be used as a marker for the induction and initiation of autophagy. Over time, the levels of phosphorylated 4E-BP1 became lower in temsirolimus-treated Nara-H cells than in control cells, but there was no difference in total 4E-BP1 protein levels between the groups at any time-point. Based on the result of this time-course analysis, we concluded that a temsirolimus treatment of $90 \mathrm{~min}$ was sufficient for all further experiments. Moreover, this timecourse analysis demonstrated that autophagy, as determined by LC-3II expression, was induced within 30 min of adding temsirolimus (Fig. 2A).

Figure 2. Western blot analysis was used to investigate the effects of temsirolimus on components of the Akt/mTOR pathway. (A) Temsirolimus caused time-dependent decreases in 4E-BP1 phosphorylation and time-dependent and dose-dependent increases in LC-3II expression. (B) Phospho-p70S6K and phospho-4EBP1 expression levels were decreased by temsirolimus treatment in a dose-dependent manner. These results indicated that temsirolimus affected mTOR and the downstream effectors by inhibiting phosphorylation. (C) In cells treated with temsirolimus or with temsirolimus and 3-MA, PARP was cleaved from the inactive $116 \mathrm{kDa}$ form to the active $89 \mathrm{kDa}$ form. This result indicated that, 3-MA can increase apoptotic cell death of Nara-H cells.

We also examined Akt, phospho-Akt, mTOR, phosphomTOR, 4E-BP1, phospho-4E-BP1, p70S6K, phospho-p70S6K, and LC-3 expression in Nara-H cells exposed to different concentration of temsirolimus (ranging from 1 to $50 \mu \mathrm{M}$ ) for 90 min (Fig. 2B). Temsirolimus did not cause any change in the levels of phospho-Akt and phospho-mTOR. However, temsirolimus treatments resulted in dose-dependent decreases in both phospho-4EBP1 and phospho-p70S6K and p7OS6K, like 4EBP1, is a downstream effector of mTOR. These findings indicate that temsirolimus affects the mTOR pathway by 

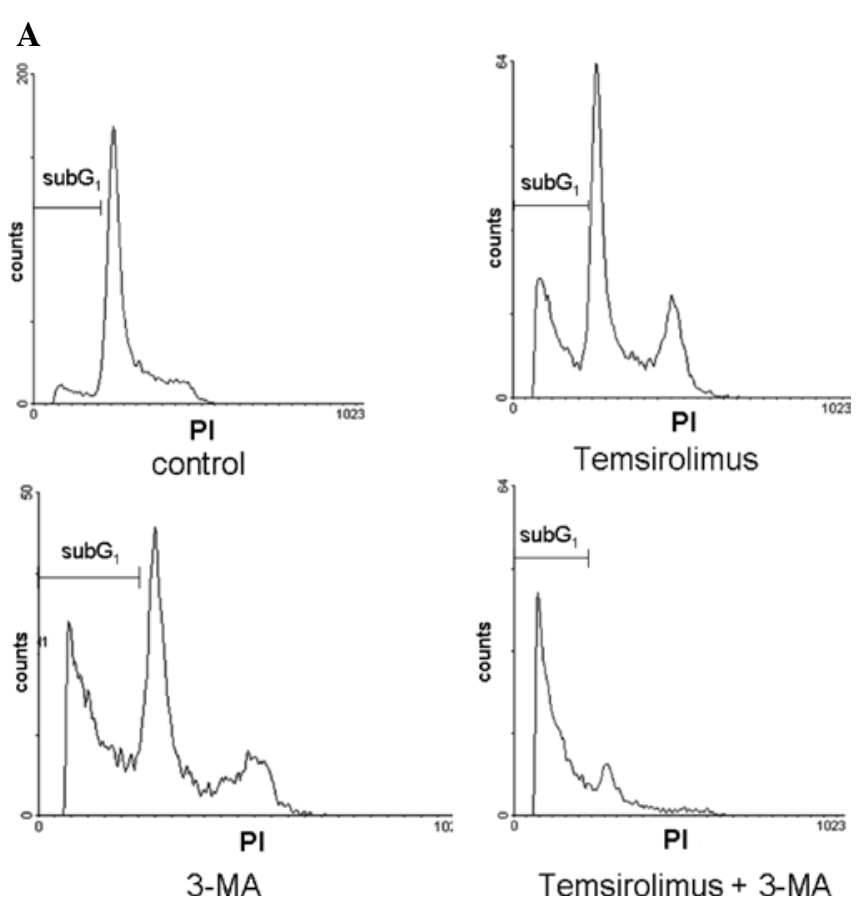

Temsirolimus

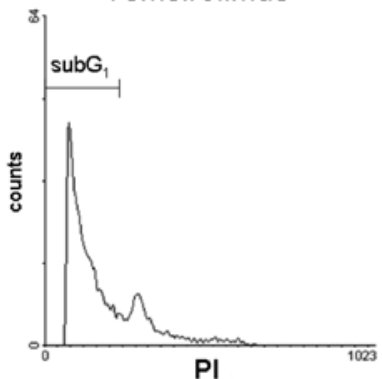

Temsirolimus + 3-MA
B

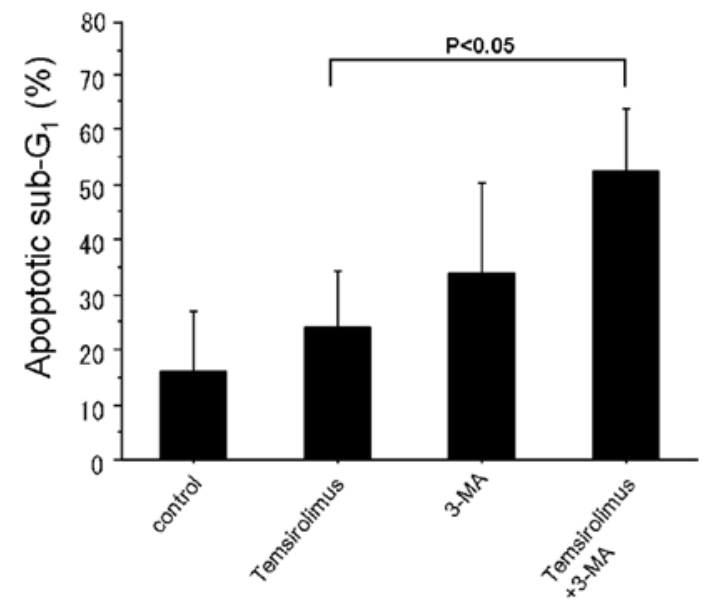

Figure 3. (A) The effect of temsirolimus with or without 3-MA on cell cycle fraction was evaluated by flow cytometry. In this study, a sub- $\mathrm{G}_{1}$ DNA content was used as a marker for apoptosis. (B) The histogram allows a comparison of the percentages of cells in Apoptotic sub- $\mathrm{G}_{1}$ fraction within each treatment group. The percentages of apoptotic cells are presented as mean $\pm \mathrm{SD}$ of three experiments. The percentage of cells in the sub- $\mathrm{G}_{1}$ fraction was significantly higher in the group treated with $35 \mu \mathrm{M}$ temsirolimus and 3-MA treatment than in the other groups $(\mathrm{p}<0.05)$.

effectors by inhibiting phosphorylation of downstream effectors of mTOR. Moreover, temsirolimus treatments resulted in dosedependent increases in LC-3II expression in Nara-H cells.

Finally, we examined PARP and LC-3 expression in Nara-H cells treated with $35 \mu \mathrm{M}$ temsirolimus with or without $5 \mathrm{mM}$ 3-MA and in untreated control cells (Fig. 2C). In cells treated with temsirolimus and temsirolimus plus 3-MA treated cells, PARP was cleaved from the inactive $116 \mathrm{kDa}$ form to the active $89 \mathrm{kDa}$ form. The expression of LC-3II was clearly higher in temsirolimus-treated cells than in untreated control cells, but LC-3II levels were lower in cells treated with both temsirolimus 3-MA than in cells treated with only Temsirolimus. These results indicated that 3-MA might increase apoptotic in Nara- $\mathrm{H}$ cells by inhibiting temsirolimus-induced autophagy.
Cell cycle fraction by flow cytometry. In this study, cell cycle fractions were separated and examined by flow cytometry

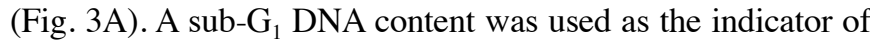
the apoptotic cell fraction. A small apoptotic fraction with a sub$\mathrm{G}_{1}$-phase content was detected in the cells treated with $35 \mu \mathrm{M}$ temsirolimus or $5 \mathrm{mM} 3$-MA. A large apoptotic fraction was detected in cells treated with $35 \mu \mathrm{M}$ temsirolimus plus $5 \mathrm{mM}$ 3-MA ( $<<0.05)$ (Fig. 3B).

Annexin V-FITEC and PI stain analysis by fluorescence micro-scopy. We used Annexin V-FITC and PI staining and fluorescence microscopy to detect apoptotic cells. Annexin V-FITC is a marker for early apoptosis, and PI is a marker for late apoptosis and necrosis. We observed several Annexin V-FITCpositive cells (early stage of apoptosis) and a great number of Annexin V-FITC plus PI positive cells (late stage of apoptosis) in Temsirolimus plus 3-MA treated group (Fig. 4A). The percentage of apoptotic cells was slightly higher in the tem group than in the control group; in contrast, the percentage of apoptotic cells was much higher in the tem-plus-3-MA group than in the controls, the 3-MA group, or the tem group (Fig. 4B).

Detection of autophagic vacuoles with monodansylcadaverine. Using fluorescence microscopy analysis, we observed fluorescence of MDC, an autofluorescent dye that is a specific marker for autophagic vacuoles, in control and treated Nara- $\mathrm{H}$ cells (19) (Fig. 5A). MDC accumulates in mature autophagic vacuoles, such as autophago-lysosomes, but not in the early endosome compartment; therefore, MDC staining is used to detect autophagic vacuoles. MDC stain analysis showed that MDC accumulation was higher and more frequent in temsirolimus-treated Nara-H cells than in control cells. These results corroborate the observation that temsirolimus treatment induces autophagy in Nara-H cells. In addition, MDC accumulation was lower and less frequent in the 3-MA group than in any of the other three groups, temsirolimus alone, temsirolimus plus 3-MA, or the control (Fig. 5B).

\section{Discussion}

Soft tissue sarcomas, especially high-grade sarcoma like MFH, are clinically aggressive and frequently metastasize to various organs. Although many chemotherapeutic protocols, including doxorubicin, cisplatin and ifosfamide, are used for human sarcomas, protocols for sarcomas are often ineffective, and the prognosis for some patients can be extremely poor due to local recurrence and/or metastasis (2). In the absence of effective systemic chemotherapy treatments for aggressive sarcomas, targeted therapies are being investigated and used as treatments. Targeted drugs, including mTOR inhibitors, are currently tested as single agents or in combination with other agents for the treatment of sarcomas $(6,20)$. Reportedly, blocking the mTOR pathway has implications for treatment of many diverse diseases (21).

Rapamycin was initially developed and given regulatory approval for the prevention of allograft rejection after organ translation because of its immunosuppressive effects. Reportedly, rapamycin and its analogues are the only known molecules that specifically bind and inhibit mTOR (22). A variety of cell signaling events in the PI3 kinase/Akt pathway are mediated by 


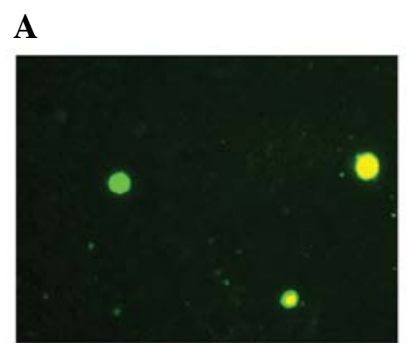

control

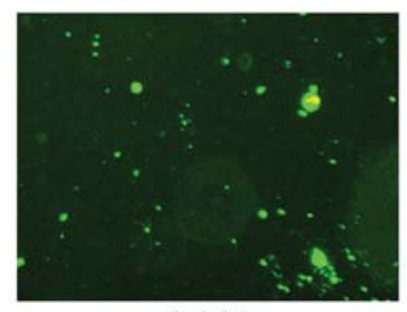

3-MA

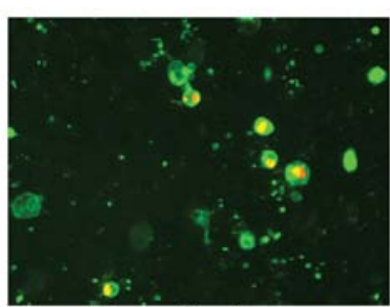

Temsirolimus

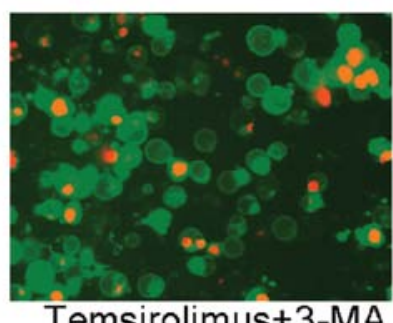

Temsirolimus+3-MA
B

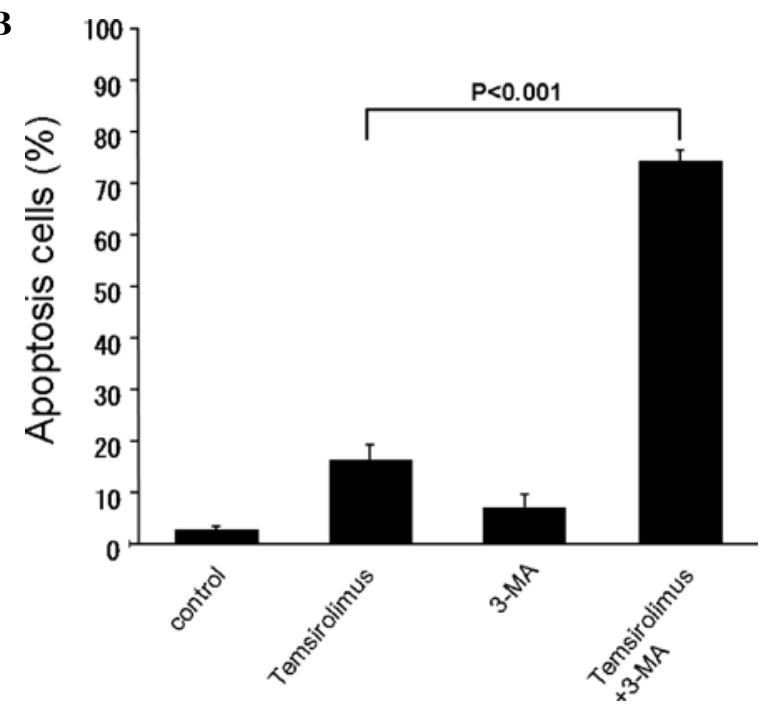

Figure 4. (A) Apoptotic cells were detected by Annexin V and PI stain. There were more Annexin V-stained cells in the group treated with temsirolimus and 3-MA than in the other groups. (B) The histogram allows a comparison of the different percentages of apoptotic cells within each treatment group. The percentages of apoptotic cells are presented as mean $\pm \mathrm{SD}$ of three experiments. The percentage of apoptotic cells was higher in the cells treated with temsirolimus and 3-MA treated than in the other groups of cells $(\mathrm{p}<0.001)$.

mTOR, and this pathway plays a central role in cell survival and proliferation in many cancers $(21,23,24)$. Abnormal activation of mTOR results in continuous activation of downstream transcription factors leading to malignant transformation. Unlike other kinases, mTOR is apparently genetically stable; it has not been shown to mutate in human malignancies. Therefore, mTOR represents a promising target for cancer therapy (6).

Once activated, mTOR phosphorylates translation-regulating factors p70S6K, a regulator of translation, and 4E-BP1, an inhibitor of eukaryote translation, to increase the synthesis of key proteins that stimulate cell growth, proliferation, and survival. Active p70S6K and active 4E-BP1 are required for and independently mediate $\mathrm{mTOR}$-dependent $\mathrm{G}_{1}$-phase progression (25). These observations indicate that mTOR inhibitors may suppress the phosphorylation of the downstream effectors, 4E-BP1 and
A

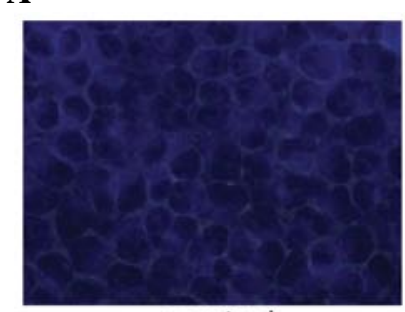

control

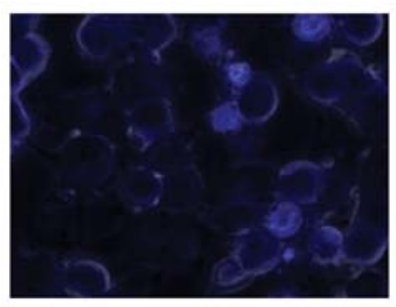

3-MA

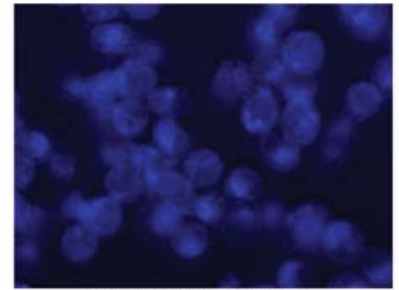

Temsirolimus

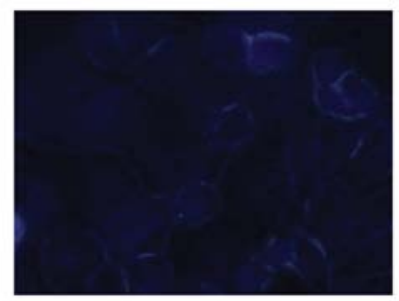

Temsirolimus+3-MA
$\mathbf{B}$

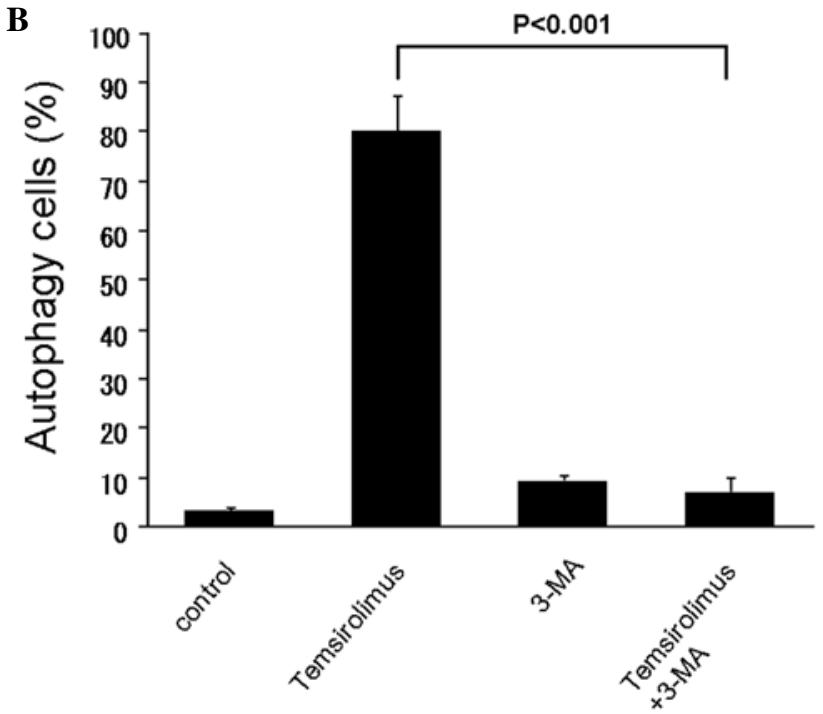

Figure 5. Autophagic cells were detected by MDC stain. Temsirolimus treatment increased the percentage of MDC-stained cells. However, treatment with temsirolimus and 3-MA decreased the percentage of MDC-stained cells relative to that in the temsirolimus-treated cells. (B) The histogram allows a comparison of the different percentages of MDC-stained cells within each treatment group. The percentages of MDC-stained cells are presented as mean \pm SD of three experiments. MDC stained cells were strongly increased in temsirolimus + 3-MA treated group $(\mathrm{p}<0.001)$.

p70S6K, and, therefore, delay progression of the cell cycle from $\mathrm{G}$ (1) phase to $S$ phase. Our data demonstrated that temsirolimus did not cause any change in phospho-Akt and phospho-mTOR protein levels. However, temsirolimus treatments resulted in dose-dependent decreases in both phospho-4E-BP1 and phospho-p70S6K, two downstream targets of mTOR.

Inhibitors of mTOR can function as inhibitors of cell proliferation that leads to cell cycle arrest or as cytotoxic agents inducing apoptosis, depending on tumor cell type (26). In these cytotoxic actions, it is important that rapamycin induces apoptosis in certain cell systems (22). Rapamycin induces apoptosis only in cells lacking functional p53, and Huang et al have shown that their results may be useful in the conceptual development of tumor-selective therapy with rapamycin analogs or other inhibitors of mTOR (27). However, some studies show that mTOR 
inhibitors induce cell death in some malignancies by autophagy rather than apoptosis $(10,28)$. Recent studies also indicate that inhibition of the Akt/mTOR pathway is consistently associated with triggering autophagy in cancer cells (9). Moreover, other studies have shown that autophagy inhibitors, such as 3-MA, induced apoptosis in some cancer cell lines when used as an anti-cancer agent in combination with an mTOR inhibitor $(14,29)$.

3-MA is an inhibitor of autophagy. However, recent reports indicate that 3-MA works well with chemotherapeutic drugs by triggering apoptosis in some cancer cells (29). In our experiment, we observed that using temsirolimus and 3-MA in combination induced more cell death in Nara-H cells than using temsirolimus alone. Therefore, it appears that together temsirolimus and 3-MA induced apoptotic cell death in Nara-H cells by inhibiting autophagy. Interestingly, our Western blots showed that LC-3II expression was higher in cells treated with a combination of temsirolimus and 3-MA than in other groups, but our fluorescence microscopy analysis of MDC-stained samples showed that there were fewer or smaller mature autophagosomes in cells treated with the temsirolimus and 3-MA combination than in other groups. Some authors report that in certain cases, though 3-MA prevents autophagy, 3-MA maintains LC-3 in its LC-3II form (30). Therefore, we suggest that while temsirolimus treatment led to LC-3II expression in Nara-H cells, the combined action of temsirolimus and 3-MA prevented actual autophagy in these cells.

Autophagy is a process in which subcellular membranes undergo dynamic morphological change (autophagosomes form and fuse with lysosomes) that lead to the degradation of cellular proteins and cytoplasmic organelles. Autophagy plays a protective role when cells encounter environmental stressors, such as starvation or pathogen infection $(31,32)$. Autophagy also occurs under pathological conditions, such as neurodegenerative disease or heredity myopathies. Recently, increasing evidence has indicated that autophagy often plays a role in malignant diseases. Specifically, autophagy is believed to have an important role in tumor development. During the early stages of tumor formation, autophagy functions as tumor suppressor, and autophagic activity is often impaired in cancer cells.

In conclusion, temsirolimus had an inhibitory effect on cell proliferation and decreased the phosphorylation of mTOR pathway components in Nara-H cells. Temsirolimus induced autophagy in Nara-H cells, but treatment with a combination of temsirolimus and 3-MA suppressed autophagy and induced apoptosis in these cells.

It was thought that the autophagy inhibitor suppressed a self-defense mechanism in tumor cells and induced apoptosis. Therefore, the combination of an mTOR inhibitor and an autophagy inhibitor (temsirolimus and 3-MA, respectively) may be an effective treatment for MFH because this combination effectively induces the apoptosis pathways.

\section{Acknowledgments}

The authors thank Mr. Kouichi Yube (Division of Research Instru-ment and Equipment, Kagawa University, School of Medecine) for his excellent technical assistance with flow cytometry.

\section{References}

1. Nakatani T, Marui T, Yamamoto T, et al: Expression of stem cell factor and c-kit in human malignant fibrous histiocytoma cell line (TNMY1). Anticancer Res 23: 2329-2333, 2003.

2. Kawamoto T, Akisue T, Kishimoto K, et al: Inhibition of PKCalpha activation in human bone and soft tissue sarcoma cells by the selective PKC inhibitor PKC412. Anticancer Res 28: 825-832, 2008

3. Wan X and Helman LJ: The biology behind mTOR inhibition in sarcoma. Oncologist 12: 1007-1018, 2007.

4. Hay N and Sonenberg N: Upstream and downstream of mTOR. Genes Dev 18: 1926-1945, 2004.

5. Gridelli C, Maione P and Rossi A: The potential role of mTOR inhibitors in non-small cell lung cancer. Oncologist 13: 139-147, 2008.

6. Mita MM and Tolcher AW: The role of mTOR inhibitors for treatment of sarcomas. Curr Oncol Rep 9: 316-322, 2007.

7. Ito D, Fujimoto $\mathrm{K}$, Mori $\mathrm{T}$, et al: In vivo antitumor effect of the mTOR inhibitor CCI-779 and gemcitabine in xenograft models of human pancreatic cancer. Int J Cancer 118: 2337-2343, 2006.

8. Easton JB and Houghton PJ: mTOR and cancer therapy. Oncogene 25: 6436-6446, 2006.

9. Kim KW, Mutter RW, Cao C, et al: Autophagy for cancer therapy through inhibition of pro-apoptotic proteins and mammalian target of rapamycin signaling. J Biol Chem 281: 36883-36890, 2006.

10. Hung JY, Hsu YL, Li CT, et al: 6-Shogaol, an active constituent of dietary ginger, induces autophagy by inhibiting the AKT/ mTOR pathway in human non-small cell lung cancer A549 cells. J Agric Food Chem 57: 9809-9816, 2009.

11. Tan ML, Ooi JP, Ismail N, Moad AI and Muhammad TS: Programmed cell death pathways and current antitumor targets. Pharm Res 26: 1547-1560, 2009.

12. Amaravadi RK and Thompson CB: The roles of therapy-induced autophagy and necrosis in cancer treatment. Clin Cancer Res 13: 7271-7279, 2007.

13. Pattingre S, Espert L, Biard-Piechaczyk M and Codogno P: Regulation of macroautophagy by mTOR and Beclin 1 complexes. Biochimie 90: 313-323, 2008.

14. Liu D, Yang Y, Liu Q and Wang J: Inhibition of autophagy by 3-MA potentiates cisplatin-induced apoptosis in esophageal squamous cell carcinoma cells. Med Oncol (In press).

15. Kanematsu S, Uehara N, Miki H, et al: Autophagy inhibition enhances sulforaphane-induced apoptosis in human breast cancer cells. Anticancer Res 30: 3381-3390, 2010.

16. Ren Y, Huang F, Liu Y, Yang Y, Jiang Q and Xu C: Autophagy inhibition through PI3K/Akt increases apoptosis by sodium selenite in NB4 cells. BMB Rep 42: 599-604, 2009.

17. Petiot A, Ogier-Denis E, Blommaart EF, Meijer AJ and Codogno P: Distinct classes of phosphatidylinositol 3'-kinases are involved in signaling pathways that control macroautophagy in HT-29 cells. J Biol Chem 275: 992-998, 2000.

18. Kiyozuka Y, Nakagawa H, Uemura Y, et al: Novel cell lines established from a human myxoid malignant fibrous histiocytoma arising in the uterus. Cancer Genet Cytogenet 127: 7-15, 2001.

19. Biederbick A, Kern HF and Elsasser HP: Monodansylcadaverine (MDC) is a specific in vivo marker for autophagic vacuoles. Eur J Cell Biol 66: 3-14, 1995.

20. MacKenzie AR and von Mehren M: Mechanisms of mammalian target of rapamycin inhibition in sarcoma: present and future. Expert Rev Anticancer Ther 7: 1145-1154, 2007.

21. Hartford CM and Ratain MJ: Rapamycin: something old, something new, sometimes borrowed and now renewed. Clin Pharmacol Ther 82: 381-388, 2007.

22. Albanell J, Dalmases A, Rovira A and Rojo F: mTOR signalling in human cancer. Clin Transl Oncol 9: 484-493, 2007.

23. Ma WW and Jimeno A: Temsirolimus. Drugs Today 43: 659-669, 2007.

24. Peponi E, Drakos E, Reyes G, Leventaki V, Rassidakis GZ and Medeiros LJ: Activation of mammalian target of rapamycin signaling promotes cell cycle progression and protects cells from apoptosis in mantle cell lymphoma. Am J Pathol 169: 2171-2180, 2006.

25. Figlin RA: Mechanisms of disease: survival benefit of temsirolimus validates a role for mTOR in the management of advanced RCC. Nat Clin Pract Oncol 5: 601-609, 2008.

26. Johnsen JI, Segerstrom L, Orrego A, et al: Inhibitors of mammalian target of rapamycin downregulate MYCN protein expression and inhibit neuroblastoma growth in vitro and in vivo. Oncogene 27: 2910-2922, 2008. 
27. Huang S, Shu L, Dilling MB, et al: Sustained activation of the JNK cascade and rapamycin-induced apoptosis are suppressed by p53/p21(Cip1). Mol Cell 11: 1491-1501, 2003.

28. Yazbeck VY, Buglio D, Georgakis GV, et al: Temsirolimus downregulates p21 without altering cyclin D1 expression and induces autophagy and synergizes with vorinostat in mantle cell lymphoma. Exp Hematol 36: 443-450, 2008.

29. Nishikawa T, Tsuno NH, Okaji Y, et al: Inhibition of autophagy potentiates sulforaphane-induced apoptosis in human colon cancer cells. Ann Surg Oncol 17: 592-602, 2010
30. Gimenez-Xavier P, Francisco R, Platini F, Perez R and Ambrosio S: LC3-I conversion to LC3-II does not necessarily result in complete autophagy. Int J Mol Med 22: 781-785, 2008.

31. Klionsky DJ and Emr SD: Autophagy as a regulated pathway of cellular degradation. Science 290: 1717-1721, 2000.

32. Meijer AJ and Codogno P: Regulation and role of autophagy in mammalian cells. Int J Biochem Cell Biol 36: 2445-2462, 2004. 